\title{
ESTUDIO DEL DESEMPEÑO ANTE LA CORROSIÓN DE LOS ACEROS N80, L80, L304, EN MEDIOS AGRESIVOS $\mathrm{CO}_{2} \mathrm{Y} \mathrm{Cl}$
}

\section{PERFORMANCE STUDY TO CORROSION OF STEEL N80, L80, L304, AGGRESSIVE MEDIA $\mathrm{CO}_{2}$ AND Cl}

\author{
Diego Figueredo-Amaya ${ }^{1} \mid$ César Armando Ortiz-Otálora ${ }^{2} \mid$ Enrique Vera-López $^{3} \mid$ Juan Pablo Vega-Triana ${ }^{4}$
}

Forma de citar: FIGUEREDO-AMAYA Diego, ORTIZ-OTÁLORA César, et al,. Estudio del desempeño ante la corrosión de los aceros N80, 180, 1304, en medios agresivos co2 y cl. Respuestas. 2013; 18(2): 80-86.

Recibido:

Marzo 3 de 2013

Aceptado:

Julio 25 de 2013

80

\section{RESUMEN}

Debido a que la gran parte de las fallas en los sistemas de levantamiento son debidos a corrosión y teniendo en cuenta los costos en los que se incurre debido a este fenómeno, se presenta la evaluación electroquímica de los aceros N80, L80, L304 usados en fondo de pozo en la industria petrolera, con la simulación de las condiciones de fondo de pozo, evaluando el efecto de la presencia y ausencia de $\mathrm{CO}_{2}$, en ambientes de cloruros. La caracterización electroquímica se realizó mediante curvas de polarización Tafel y resistencia a la polarización lineal (LPR), las cuales permitieron encontrar que la menor resistencia a la polarización se presenta en el acero N80, para condiciones dinámicas de $\mathrm{pH}(7)$, temperatura de $70{ }^{\circ} \mathrm{C}$ y presión de 300 psi.

Palabras clave: electroquímica, API N80, API L80, API L304, tafel, fondo de pozo.

\section{ABSTRACT}

Universidad Pedagógica y Tecnológica de Colombia. dfigueredoa82@gmail.com Tunja - Colombia

${ }^{2}$ Magister en Ciencias Física Universidad Pedagógica y Tecnológica de Colombia. cesar.ortiz@uptc.edu.co Tunja-Colombia

${ }^{3}$ Magister en Física Universidad Pedagógica y Tecnológica de Colombia vera@uptc.edu.co Tunja - Colombia

${ }^{4}$ Magister en Metalurgia y Ciencia de Materiales Universidad Pedagógica y Tecnológica de Colombia Tunja - Colombia
Because much of the system failures are due to rising corrosion and taking into account the costs incurred due to this phenomenon, We had to compare and evaluate the reaction of three kinds of steel used in the oil industry, based on the simulation of the conditions at the downhole, evaluating the effect of presence or absence of $\mathrm{CO}_{2}$ in an environment showing chlorides. The electrochemical characterization was conducted by means of polarization graphs (Tafel) and graphs showing the resistance to linear polarization (LPR). As a result, the least polarization occurred in N80 steel under dynamic conditions $(\mathrm{pH} 7)$ at $70^{\circ} \mathrm{C}$ and under a 300 psi pressure.

Keywords: electrochemical, API N80, API L80, API L304, Tafel, downhole. 


\section{INTRODUCCIÓN}

$\mathrm{D}$ urante los procesos de completamiento y producción de los pozos petroleros se presentan fenómenos de corrosión debido a las condiciones especialmente agresivas que se presentan en estos medios, lo que conlleva a que de la mayoría de fallas que ocurren en la industria de gas y petróleo, la corrosión sea una de las más significativas; ocasionando pérdidas que pueden llegar a cifras muy importantes $[1,2]$.

La mayoría de los equipos utilizados en la industria están constituidos de metal, estos al estar dentro del pozo, son susceptibles de sufrir procesos de corrosión debido a la presencia de gases disueltos como el $\mathrm{CO}_{2}$ y cloruros, estas son las especies que contribuyen principalmente a los fenómenos de corrosión y contra las cuales es necesario actuar [2-8].

Teniendo en cuenta los costos que conllevan una falla por corrosión, debido entre otros factores a una posible mala elección de material, mediante este trabajo se pretende contar con una guía que permita seleccionar el tubing adecuado según las condiciones fisicoquímicas a las que va a ser expuesto disminuyendo de esta manera las fallas por corrosión de este elemento [9-11].

\section{MATERIALES Y MÉTODOS}

Para la evaluación se emplearon piezas mecanizadas cilíndricas de $10 \mathrm{~mm}$ de diámetro por $14 \mathrm{~mm}$ de altura que se toma de una sección de tuberías identificadas como acero API N80, API L80 y API L304. Para garantizar que el material utilizado en las pruebas correspondía con los aceros API se les realizó la caracterización composicional por espectroscopia de chispa presentados en las Tablas 1 .

Adicionalmente se prepararon probetas de los aceros para la caracterización electroquímica de acuerdo con las dimensiones del equipo (Figura 1). Para determinar la influencia de cada una de las variables estudiadas como: flujo estático o dinámico, presión de 300 o 500 Psi, solución salina $(\mathrm{NaCl}$ al $3.5 \%)$ con y $\sin \mathrm{CO}_{2}$, con un $\mathrm{pH}$ de 7 a temperatura constante de $70^{\circ} \mathrm{C}$. Se realizó un control paramétrico que consiste; en mantener constante cada variable y realizando modificaciones a las restantes, hasta encontrar las combinaciones posibles.

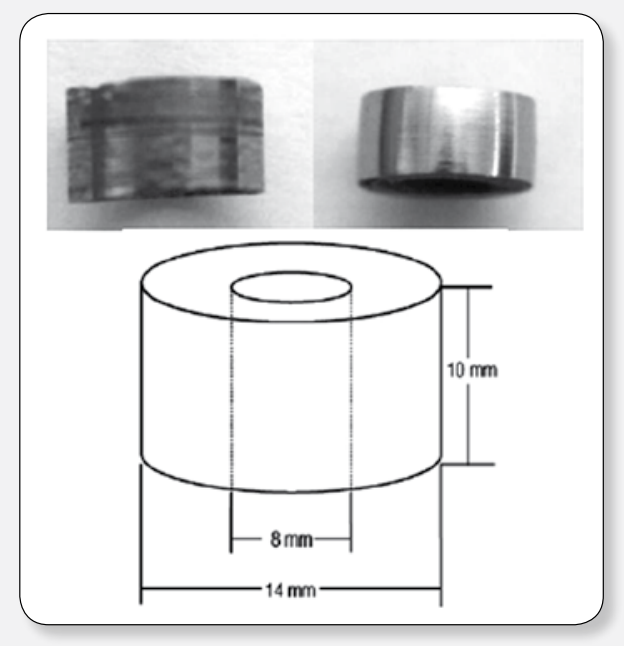

Figura 1. Preparación superficial y dimensiones de las probetas.

Fuente: Autor

Tabla 1. Composición química aceros N80, L80, L304.

\begin{tabular}{|c|c|c|c|}
\hline \multicolumn{4}{|c|}{ COMPOSICIÓN QUÍMICA ACEROS } \\
\hline Elemento & N80 (\%) & L80 (\%) & L304 (\%) \\
\hline $\mathrm{Al}$ & 0.0547 & 0.0259 & NR \\
\hline $\mathrm{C}$ & 0.164 & 0.321 & 0,035 \\
\hline $\mathrm{Co}$ & $<0,00500$ & $<0.00501$ & NR \\
\hline $\mathrm{Cr}$ & 0.00500 & 0.965 & 18.0 a 20.0 \\
\hline $\mathrm{Cu}$ & 0.0372 & 0.0812 & NR \\
\hline $\mathrm{Fe}$ & 97.9 & 97.6 & NR \\
\hline $\mathrm{Mn}$ & 1.51 & 0.511 & 2.0 \\
\hline $\mathrm{Mo}$ & $<0.00510$ & 0.186 & NR \\
\hline $\mathrm{Nb}$ & 0.00245 & 0.00346 & NR \\
\hline $\mathrm{Ni}$ & 0.0156 & 0.0272 & 8.0 a 13.0 \\
\hline $\mathrm{P}$ & 0.0113 & 0.0144 & 0.04 \\
\hline $\mathrm{S}$ & 0.00880 & 0.00638 & 0.03 \\
\hline $\mathrm{Si}$ & 0.209 & 0.240 & 0.75 \\
\hline $\mathrm{SN}$ & 0,00245 & 0.00431 & NR \\
\hline $\mathrm{TI}$ & 0.00106 & 0.00201 & NR \\
\hline $\mathrm{V}$ & 0.00445 & 0.00395 & NR \\
\hline $\mathrm{W}$ & 0.0168 & 0.0180 & NR \\
\hline Nota: NR no reporta & & \\
\hline
\end{tabular}

Fuente: Autor.
Junio - Diciembre 2013 ISSN 0122-820X

PP: $80-86$

\section{1}


No. 2

Junio - Diciembre 2013 ISSN 0122-820X

PP: $80-86$
Para realizar un adecuado control paramétrico, que permita realizar la totalidad de pruebas y combinaciones, se construyó la matriz de pruebas para $\mathrm{pH}$ de 7 y temperatura $70^{\circ} \mathrm{C}$. Para cada acero (L-80, N-80, y L-304), se usó una solución de trabajo $\mathrm{NaCl}$ al 3.5\%, la cual simula las condiciones de mayor agresividad de un pozo petrolero, en atmosferas con y sin $\mathrm{CO}_{2}$, en condiciones estáticas y dinámicas a presiones de 300 y 500 psi [14].

Pruebas electroquímicas. Para la realización de las pruebas se utilizó un reactor de alta presión y alta temperatura, acoplado a un potenciostato-galvanostato marca GAMRY INSTRUMENTS SERIE G 300 (Figura 2). Las probetas son piezas cilíndricas de acero tipo API N80, API L80, API L304 según la (Figura 1). El montaje básico de una prueba consiste en la preparación de la solución con: agua destilada y adición de ácido nítrico o cloruro de sodio (salmuera al 3,5\% de $\mathrm{NaCl}$ ), de acuerdo al valor requerido, el montaje final se esquematiza en la Figura 2

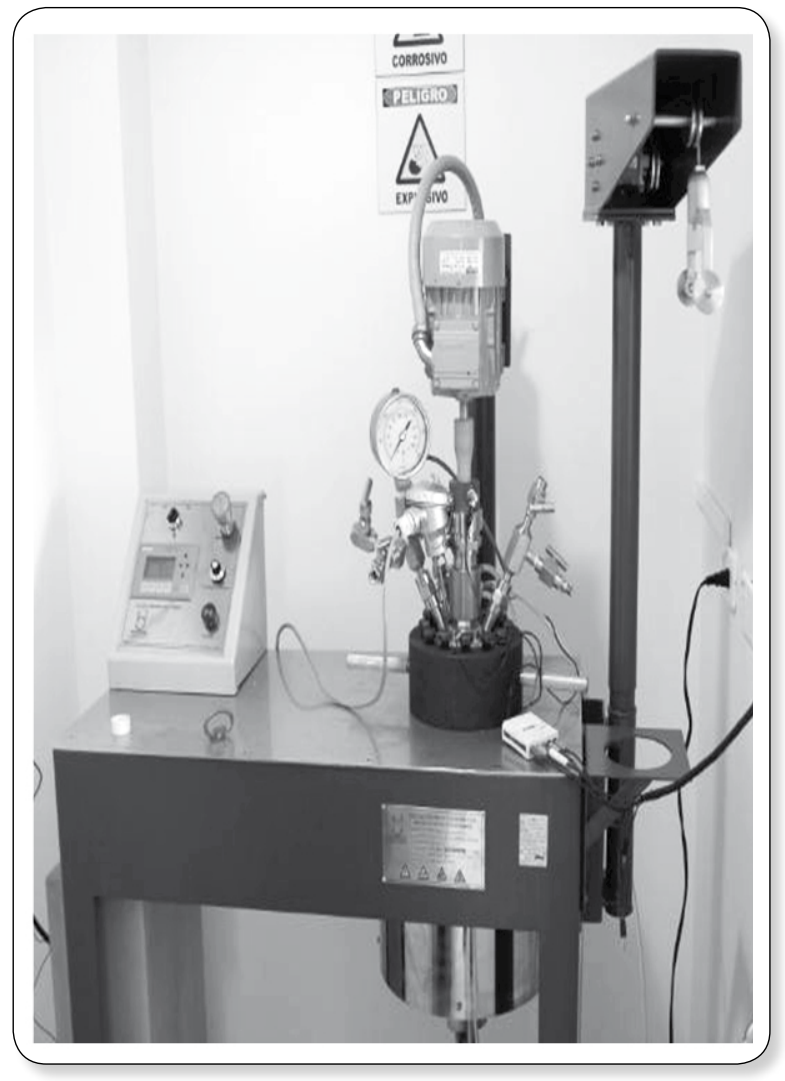

Figura 2. Reactor de alta presión y temperatura

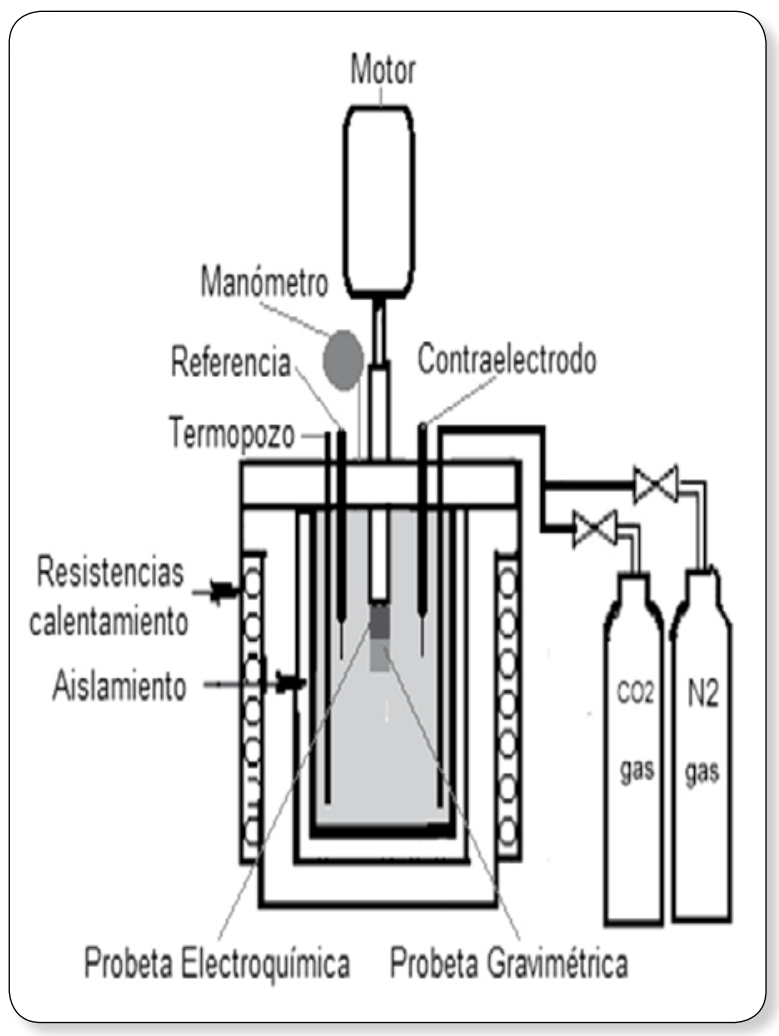

Fuente: Autor.

La solución de prueba se introduce en el recipiente del reactor, debido a que los sistemas de pozos petroleros tienen concentraciones muy bajas o nulas de oxígeno, se realiza la apertura de la válvula de ingreso de $\mathrm{N}_{2}$ al reactor, se mantiene el paso de gas durante 1 hora para eliminar el oxígeno presente en el recipiente, luego de la remoción de oxígeno se realiza la inyección de $\mathrm{CO}_{2}$ hasta el valor requerido por la prueba, finalmente se introduce $\mathrm{N}_{2}$ hasta alcanzar 1250 psi en todas las pruebas, para esto el equipo cuenta con un manómetro analógico y un sensor de presión. Luego de 45 minutos de estabilización de potencial se realiza la medición de resistencia a la polarización lineal $(-20 \mathrm{a}+20 \mathrm{mV})$ y la curva de polarización Tafel $(-250$ hasta $250 \mathrm{mV})$ dadas con variables adicionales como temperatura de $70^{\circ} \mathrm{C}$ y velocidad de flujo de $0,5 \mathrm{~m} / \mathrm{s}$; en la Figura 3 se muestra como ejemplo una prueba realizada a una de las muestras objeto del estudio (acero L304). 


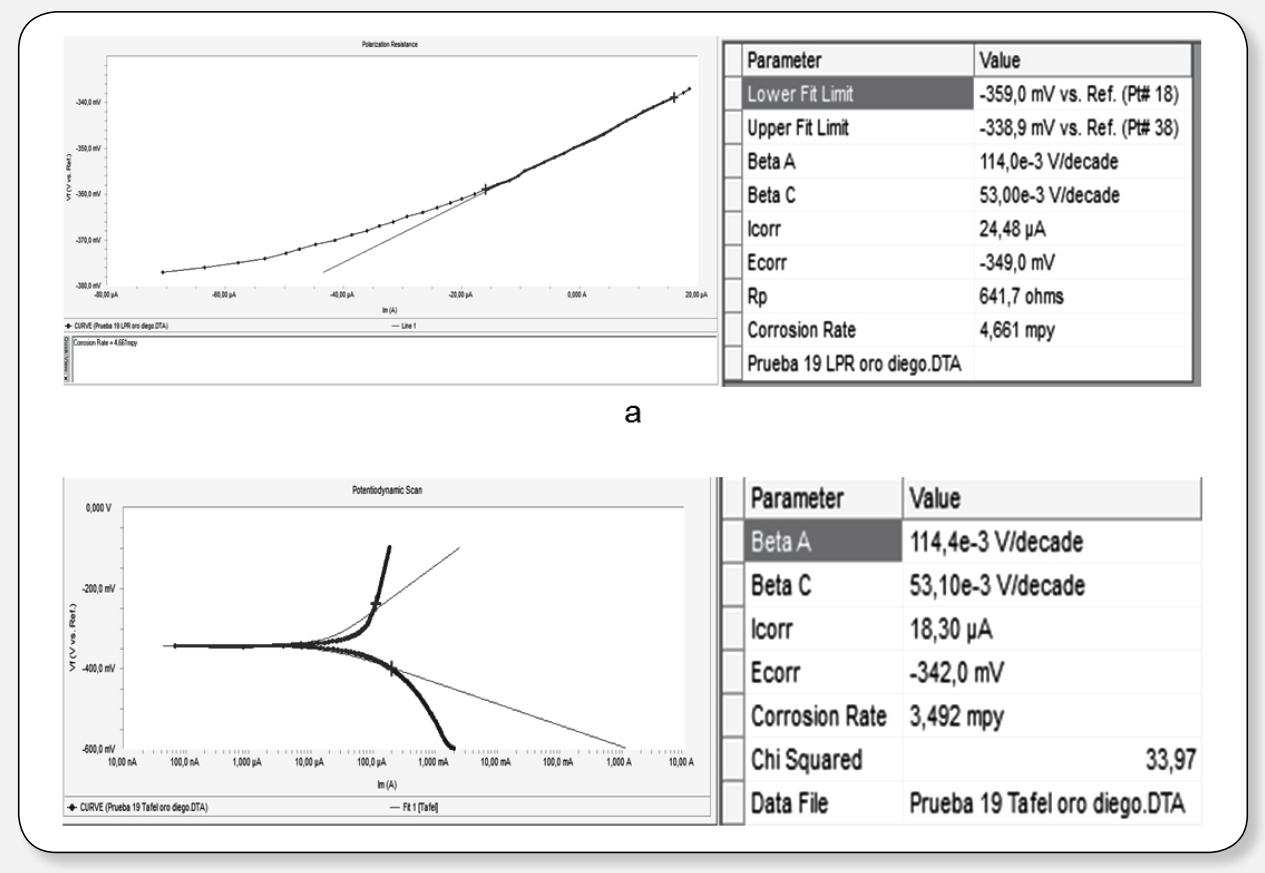

Junio - Diciembre 2013

ISSN 0122-820X

PP: $80-86$

Figura 3. a) Resistencia a la polarización lineal, b) Curva de polarización tafel.

Fuente: Autor.

En el procedimiento experimental de las técnicas electroquímicas se utilizó el montaje de tres electrodos, conformado por el electrodo de trabajo, el electrodo de referencia y el contraelectrodo de oro de alta pureza; luego las probetas seleccionadas se retiran de los recipientes de almacenamiento y son analizadas.

\section{RESULTADOS}

Los valores de resistencia a la polarización obtenidos y presentados en la Tabla 2 y 3 muestran un comportamiento del mismo orden de magnitud para cada una de las variables en las dos técnicas LPR y TAFEL.

Tabla 2. Resistencia a la polarización $(\Omega)$ por TAFEL y LPR en condiciones Estáticas.

\begin{tabular}{|c|c|c|c|c|c|}
\hline \multirow{2}{*}{$\begin{array}{l}\text { MUESTRA } \\
\text { C. Estáticas }\end{array}$} & \multirow{2}{*}{$\begin{array}{c}\text { PRESIÓN } \\
\text { Psi }\end{array}$} & \multicolumn{2}{|c|}{ LPR (Ohm) } & \multicolumn{2}{|c|}{ TAFEL (Ohm) } \\
\hline & & $\operatorname{Sin} \mathrm{CO}_{2}$ & Con $\mathrm{CO}_{2}$ & $\operatorname{Sin} \mathrm{CO}_{2}$ & Con $\mathrm{CO}_{2}$ \\
\hline \multirow{4}{*}{$\mathrm{N}-80$} & \multirow{2}{*}{300} & 123,9 & & 120,4 & \\
\hline & & & 11,7 & & 8,0 \\
\hline & \multirow{2}{*}{500} & 123,2 & & 169,1 & \\
\hline & & & 27,3 & & 9,1 \\
\hline \multirow{4}{*}{ L-80 } & \multirow{2}{*}{300} & 152,3 & & 278,6 & \\
\hline & & & 9,4 & & 10,4 \\
\hline & \multirow{2}{*}{500} & 445 & & 368,9 & \\
\hline & & & 9,8 & & 8,9 \\
\hline \multirow{4}{*}{ L-304 } & \multirow{2}{*}{300} & 928,6 & & 1942,2 & \\
\hline & & & 641,7 & & 1087,6 \\
\hline & \multirow{2}{*}{500} & 1329,0 & & 2084,7 & \\
\hline & & & 1390,0 & & 1308,6 \\
\hline
\end{tabular}

Fuente: Autor. 
Tabla 3. Resistencia a la polarización por TAFEL y LPR en condiciones dinámicas.

\begin{tabular}{|c|c|c|c|c|c|}
\hline \multirow{2}{*}{$\begin{array}{l}\text { MUESTRA } \\
\text { C. Dinámica }\end{array}$} & \multirow[t]{2}{*}{ PRESIÓN } & \multicolumn{2}{|c|}{ LPR } & \multicolumn{2}{|c|}{ TAFEL } \\
\hline & & $\operatorname{Sin} \mathrm{CO}_{2}$ & $\mathrm{Con} \mathrm{CO}_{2}$ & $\operatorname{Sin} \mathrm{CO}_{2}$ & $\mathrm{Con} \mathrm{CO}_{2}$ \\
\hline \multirow{4}{*}{$\mathrm{N}-80$} & \multirow{2}{*}{300} & 138,7 & & 159,3 & \\
\hline & & & 3,4 & & 3,2 \\
\hline & \multirow{2}{*}{500} & 27,4 & & 30,9 & \\
\hline & & & 13,0 & & 11,7 \\
\hline \multirow{4}{*}{ L-80 } & \multirow{2}{*}{300} & 71,3 & & 145,4 & \\
\hline & & & 12,9 & & 8,9 \\
\hline & \multirow{2}{*}{500} & 25,5 & & 29,0 & \\
\hline & & & 16,7 & & 16,3 \\
\hline \multirow{4}{*}{ L-304 } & \multirow{2}{*}{300} & 765,4 & & 1051,9 & \\
\hline & & & 685,1 & & 2102,0 \\
\hline & \multirow{2}{*}{500} & 1144,0 & & 832,7 & \\
\hline & & & 1038,0 & & 1449,7 \\
\hline
\end{tabular}

Fuente: Autor.

En el sistema sin $\mathrm{CO}_{2}$, se observa que los tres materiales presentan un comportamiento acorde a sus características anticorrosivas (contenido de cromo). La presión de $\mathrm{CO}_{2}$ influye en las propiedades anticorrosivas, indicando que a mayor velocidad del fluido mayor corrosión.

En los sistemas saturados con $\mathrm{CO}_{2}$ se observa una disminución importante en la resistencia a la polarización lo cual se ve reflejado en un aumento considerable de la velocidad de corrosión.

Los resultados nos muestran que la presencia de $\mathrm{CO}_{2}$ contribuye con el proceso de corrosión lo que implica un aumento en las propiedades conductoras de la interface acero fluido y la consecuente disminución de la Rp.

En los sitemas evaluados a menor presión (300 psi) se evidencia una menor Rp que los sistemas a 500 psi, esto nos indica que bajo estas condiciones el acero con mayor tendencia a la corrosión es el acero N80 a 300 psi. Resultados que concuerdan con los obtenidos en trabajos similares [12,14].

En el sistema sin $\mathrm{CO}_{2}$, se evidencia nueva- mente que los tres materiales presentan un comportamiento acorde a sus características anticorrosivas (contenido de cromo), siendo el acero L 304 el que presenta mayor Rp, adicionalmente se observa como las condiciones dinámicas del sistema afectan los valores de $\mathrm{Rp}$ haciendo que se presente menores valores que los encontrados para condiciones estáticas.

Con presencia de $\mathrm{CO}_{2}$ dadas las condiciones dinámicas del sistema, las cuales favorecen el desarrollo de reacciones electroquímicas en la superficie del metal, se presenta aún menor $\mathrm{Rp}$ dada la saturación con este compuesto.

La Rp del N80 es menor que la Rp del L80 y a su vez menor que la Rp del L304, esto implica que el acero N80 en condiciones dinámicas y a presión de 300 psi presenta la más baja $\mathrm{Rp}$ por lo cual presenta la velocidad de corrosión más alta.

Si se tiene en cuenta inicialmente que el $\mathrm{CO}_{2}$ se hidrata mediante la reacción con el agua para producir ácido carbónico $\left(\mathrm{H}_{2} \mathrm{CO}_{3}\right)$, donde sufre una doble disociación dando formación de iones carbonato y bicarbonato, permi- 
tiendo llevar un trasporte de masa desde la solución hacia la superficie del metal para que se desarrollen las reacciones electroquímicas (catódica y anódica) en la cual las especies disueltas se combinan y forman el carbonato de hierro $\left(\mathrm{FeCO}_{3}\right)$ [14,15], el cual es retirado por el fluido.

Comparando las $\mathrm{Rp}$ en condiciones estáticas y dinámicas el comportamiento es similar en magnitud tanto para la presencia de $\mathrm{CO}_{2}$ como para sin $\mathrm{CO}_{2}$ (Figura 4).

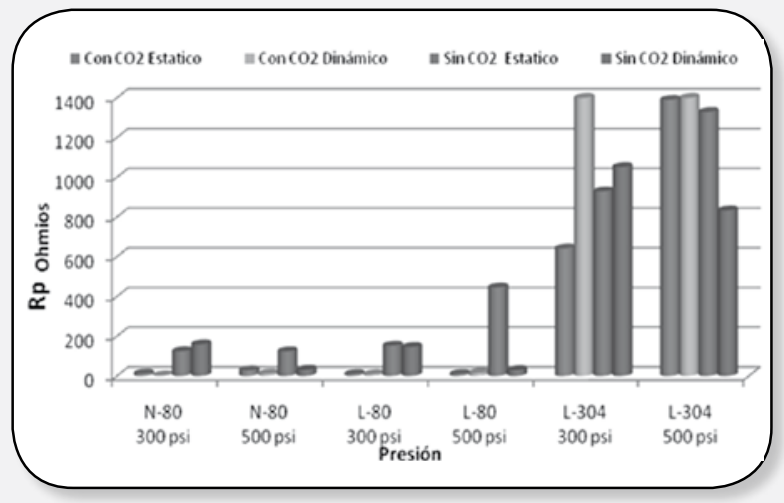

Figura 4. Resistencia a la polarización de los sistemas evaluados.

Fuente: Autor.

\section{CONCLUSIONES}

Según el análisis de los resultados de la evaluación electroquímica se concluye que la velocidad de corrosión aumenta para el sistema acero-electrolito por la presencia de $\mathrm{CO}_{2}$ y cloruros, especialmente en el acero N80 debido a que este acero es el que presenta menor contenido $\mathrm{Cr}$.

Influencia de la presión de $\mathrm{CO}_{2}$ : Se observó que este influye en las propiedades anticorrosivas de los aceros evaluados, indicando que a mayor velocidad del fluido mayor corrosión.

Las pruebas electroquímicas (LPR y TAFEL) revelan que las resistencias a la corrosión de los aceros N80, L80 y L304 con medios de $\mathrm{CO} 2, \mathrm{Cl}$ son equivalentes.

\section{AGRADECIMIENTOS}

A Juan Pablo Vega Triana, por su valiosa colaboración en el desarrollo de las pruebas. A la UPTC por facilitar la logística para el desarrollo de esta investigación. Al INCITEMA por toda la colaboración brindada.

\section{REFERENCIAS}

[1]. Metals handbook. Corrosion: Fundamental, Testing and Protection. En: kinetics of aqueouscorrosion. Vol. 13.p. 32.

[2]. Guerra U, Espinosa S, Espinoza M. Corrosión formas y control en un campo petrolero. 1991.

[3] CROLET, J.L; THEVENOT, $\mathrm{N}$ and DUGSTAD, A. Role of Free Acetic Acid on the $\mathrm{CO}_{2}$ Corrosion of Steels. En: Corrosion 99, paper no. 24. NACE, 1999.

[4] KERMANI. M.B. and SMITH, L.M. Smith. CO2 Corrosion Control in Oil and Gas Production-Design Considerations, European Federation of Corrosion, vol. 23, London, U.K.: Institute of Materials, p 660. 1997.

[5]. DALAYAN E, et al. CO2 corrosion prediction in pipe flow under $\mathrm{FeCO}_{3}$ scale-forming conditions. En: Corrosion, paper 51. 1998.

[6]. KERMANI, M.B. and SMITH, L.M. Smith. CO2 Corrosion Control in Oil and Gas Production-Design Considerations, European Federation of Corrosion, vol. 23, London, U.K.: Institute of Materials, 1997.

[7]. CROLET, J.L. - Which $\mathrm{CO}_{2}$ Corrosion, Hence Which Prediction?, Predicting $\mathrm{CO}_{2}$ Corrosion in the Oil and Gas Industry, European Federation of
Junio - Diciembre 2013 ISSN 0122-820X

PP: $80-86$ 
No. 2

Junio - Diciembre 2013 ISSN 0122-820X

PP: $80-86$
Corrosion, vol. 13. London, U.K.: Institute of Materials, 1994, p. 1.

[8]. Ortiza C, Keitelmanb A. Analisis De Falla De Un Tubo De Pozo Petrolero De Circuito De Recuperacion Secundaria En Un Yacimiento De Comodoro Rivadavia. JORNADAS SAM/ CONAMET/ SIMPOSIO MATERIA. 2003.

[9]. VIDEM, K and KOREN, A.M. En: Corrosion, vol. 9, 1993, p. 746-754.

[10]. CROLET, J.L; THEVENOT, $\mathrm{N}$ and NESIC, S. Role of Conductive Corrosion Products on the Protectiveness of Corrosion Layers. En: Corrosion, paper no. 4, 1996.

[11]. CROLET, J.L; BERANGER, G and MAZILLE, $\mathrm{H}$. Corrosion in Oil and Gas Production. En: Corrosion and Anticorrosion. 2002.

[12]. Urbáez R., Gonzalea F. Estudio Integral de la Corrosión en pozos petroleros intervenidos en la campaña 2007-2008 en el campo Uracoa, empresa mixta petrolera. 2009.

[13]. GONCALVES REIS A., Selección de Inhibidores de Corrosión para Aplicaciones en Fondo de Pozos con Levantamiento Artificial por Bombeo Electrosumergible. 2008.

[14]. VEGA J.P. Estudio Paramétrico De Variables De Corrosión En Fluidos Acuosos Que Contienen CO . UPTC. 2013.

[15] FERNÁNDEZ M. Corrosión en la industria petrolera. Universidad de Zulia. 2011. 